\title{
Granular Cell Tumor of the Breast: Clinical Presentation, Pathological Diagnosis and Treatment
}

Scardina $L^{1 *}$, Di Leone A ${ }^{1}$, Sanchez AM1, D'Archi $S^{1}$, Biondi E', Carnassale B', $D^{\prime}$ Alessandris $N^{2}$, Scaglione $G^{2}$, Santoro $A^{2}$, Mulè $A^{2}$, Masetti $R^{1}$ and Franceschini G

${ }^{1}$ Centro Integrato di Senologia, Dipartimento Scienze della Salute della Donna, del Bambino e di Sanità Pubblica, Fondazione Policlinico Universitario Agostino Gemelli IRCCS, Rome, Italy

${ }^{2}$ Unità di Gineco-patologia e Patologia Mammaria, Dipartimento Scienze della Salute della Donna, del Bambino e di Sanità Pubblica, Fondazione Policlinico Universitario Agostino Gemelli IRCCS, Rome, Italy

*Corresponding author: Lorenzo Scardina,

Multidisciplinary Breast Center, Fondazione Policlinico Universitario Agostino Gemelli IRCCS, Largo Agostino Gemelli 8, 00168 Rome, Italy

Received: August 12, 2021; Accepted: September 17, 2021; Published: September 24, 2021

\begin{abstract}
Introduction: Granular cell tumor is a rare neoplasm of soft tissue and only in $1 \%$ of cases, it can shows a malignant behaviour. It is presumed to be a tumor originating from perineural or putative Schwann cells of peripheral nerves.

Materials and Methods: We reviewed five patients affected by Granular cell tumor of the breast treated between January 2011 and January 2021 at the Fondazione Policlinico Universitario Agostino Gemelli IRCCS of Rome, Italy.

Results: All of the granular cell tumors presented as solitary, painless and firm lump, highly suggestive of malignancy. The radiological findings were heterogeneous and non-specific. All lesions presented as masses, more clearly evident on ultrasound as hypoechoic lesions, with irregular shape, blurred contours and borderline features.
\end{abstract}

The tumors were composed of large polygonal cells with abundant eosinophilic granular cytoplasm and small, central nuclei, being immunohistochemically positive for S100, Vimentin (with variable staining), CD56; negative for HMB45, MelanA, AE1/AE3, EMA, and Desmin.

Conclusion: Granular cell tumor is a rare, usually benign breast disease that can have very similar characteristics to breast cancer both clinically and radiologically. Treatment of choice consists in wide resection or lumpectomy with margin assessment (no ink on tumor).

Keywords: Granular cell tumor; Breast; Benign tumor

\section{Introduction}

Granular Cell Tumor (GCT) is a rare slowly growing neoplasm of soft tissue, representing $0.5 \%$ of all soft tissue tumors [1]. Tongue represent the most frequent site of origin ( $40 \%$ of cases). However, in only $1 \%$ of cases, it can shows a malignant behaviour $[2,3]$.

Generally, it appears as a single nodule, but multiple granular cell tumors may be observed in the context of LEOPARD syndrome, due to a mutation in the PTPN11 gene [4]. Between 4 and $6 \%$ of GCTs have been diagnosed in the breast and in $98 \%$ of cases they represent benign tumors, accounting for $0.1 \%$ of all cases of breast neoplasms [3]. Localization in breast tissue was described for the first time by Abrikossoff in 1926 [5].

It is presumed to be a tumor originating from perineural or putative Schwann cells of peripheral nerves or from their precursors, by growing in the context of the intralobular stroma of breast parenchyma and spreading through the cutaneous branches of the supraclavicular nerve [6-8]. Due to the clinical and instrumental (mammography and ultrasound) similarities with malignant tumors, differential diagnosis including histopathological examination and immunohistochemical studies is mandatory in order to apply the adequate treatment and follow up options [9].

GCT has been frequently diagnosed in middle-aged premenopausal women and particularly in women of Afro-American ethnicity, while it is extremely rare in male patients [8]. In about
$70 \%$ of cases it is discovered at clinical palpation, $26 \%$ of cases are identified by mammography screening and $4 \%$ during the follow-up of breast cancer [9].

In symptomatic patients it occurs in a variable way. The most frequent site is the upper inner quadrant of the breast, configuring a difference with breast cancer, which is more often found in the upper outer quadrant [10]. They are usually described as small, painless and mobile nodules. Sometimes, they are reported as painful masses of elastic consistency, anchored to the pectoral muscle. There is also great variability in the radiological presentation. The nodules can appear as well defined, regular homogeneous or irregular masses, often simulating a breast cancer. In some cases, the overlying skin appears thickened and retracted. GCT is generally not associated with lymphadenopathy [8-10].

In this paper, we reported a series of 5 patients affected by GCT of the breast observed at our Institute between January 2011 and January 2021.

\section{Materials and Methods}

All data were collected from our database at the Fondazione Policlinico Universitario Agostino Gemelli IRCCS of Rome, Italy. The diagnosis have been confirmed histologically in all cases. Preoperatively, a clinical examination and a radiological imaging study with ultrasound, digital mammography and MRI were performed, in order to evaluate any differences between GCT and classic breast 
Table 1: Patients characteristics.

\begin{tabular}{|c|c|c|c|c|c|}
\hline Age Onset & Side & Location & Size of the lesion $\mathbf{( m m})$ & Clinical features & Radiological Findings \\
\hline $\mathbf{5 2}$ & $\mathrm{R}$ & Ul/subclavicular region & 5 & Palpable Borderline \\
\hline $\mathbf{3 1}$ & $\mathrm{R}$ & Ll/transition between the lower quadrants & 7 & Non-palpable Benign \\
\hline $\mathbf{1 6}$ & $\mathrm{L}$ & LO & 8 & Palpable Borderline \\
\hline $\mathbf{5 5}$ & $\mathrm{R}$ & Transition between outer quadrants & 10 & Palpable Borderline & Borderline features \\
\hline $\mathbf{5 1}$ & $\mathrm{L}$ & LO & 14 & Palpable Benign & Suspicious appearing mass on US \\
\hline
\end{tabular}

carcinoma. Each case has been subjected to multidisciplinary discussion with radiologists, breast pathologists and breast surgeons of our team, to define the best treatment procedure.

\section{Results}

We identified five cases of GCT of the breast treated between January 2011 and January 2021. All cases reviewed occurred in women and three patients were in postmenopausal age. One of them reported known familiarity with breast cancer, and none had significant comorbidities.

All of the GCTs presented as solitary, painless and firm lumps, palpable in three cases and non-palpable in two cases. On clinical examination, no nodule was larger than $1.5 \mathrm{~cm}$ and none presented with enlarged lymph nodes. There was no nipple discharge or skin signs suspected of malignant disease. The tumors were located the left breast in two cases and in the right breast in three cases. Of the five GCTs, two were located in the lower-outer quadrant, one were located at the junction of the outer quadrants, one at the transition between the lower quadrants and one at upper-inner quadrants, almost at the subclavicular region (Table 1).

The radiological findings were heterogeneous and non-specific. All lesions presented as masses, more clearly evident on ultrasound as hypoechoic lesions, with irregular shape, blurred contours and borderline features (Figure 1).

In one case, the opacity on mammography also showed microcalcifications and spiculated, ill-defined margins. One case was not evident on mammography but presented as a lesion that progressively has doubled in volume over a year on ultrasound.

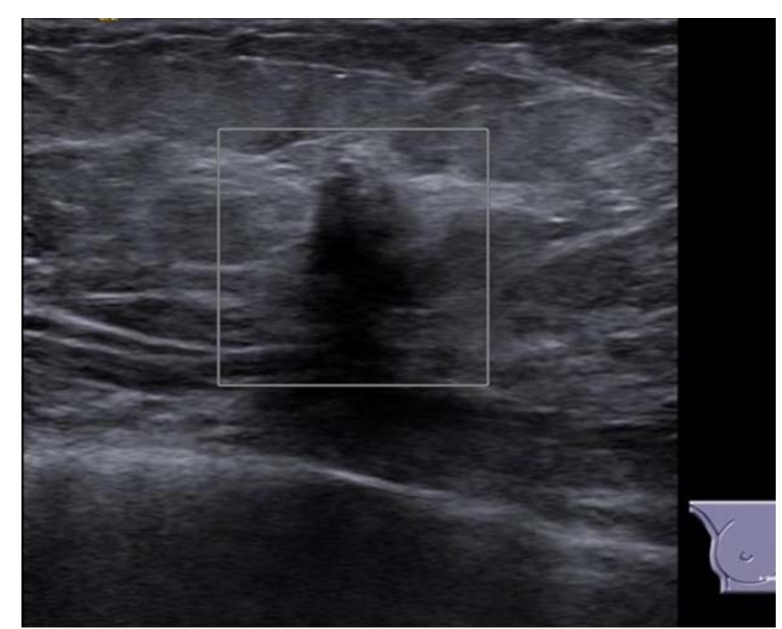

Figure 1: Ultrasound reveals a suspicious-appearing mass with posterior acoustic shadowing, not vascularized on color Doppler.

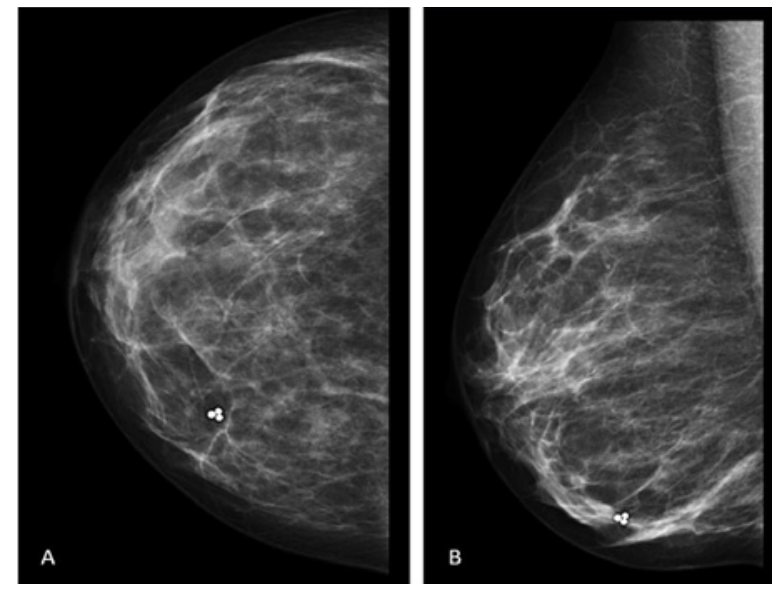

Figure 2: Cranio-caudal (A) and Mediolateral Oblique (B) mammographic view localization of non-palpable lesion.

One GCT was located superficially on ultrasound, while the others were located at different depths. Core needle biopsy procedure was performed in four cases, fine needle aspiration procedure was performed in one case, and all the lesions were classified as benign neoplasms in the preoperative and post-operative settings. Pathologically, the cytology smears showed cells clusters with moderate to abundant granular eosinophilic cytoplasm and bland nucleus.

All cases underwent surgical excision with all margins free of tumor. In one case, preoperative ultrasound-guided tattooing and mammography-guide localization of non-palpable lesions was required (Figure 2).

At the pathological examination, the 5 GCTs have a nodular appearance, greyish-white to pale yellow in colour, in three cases with a lobulated fashion, in the remaining two cases with not well circumscribed margins.

No ulceration and necrosis has been observed. Microscopically, circumscribed architecture has been reported. The nodular tumors were composed of large polygonal cells with abundant eosinophilic granular cytoplasm and small, central nuclei, being immunohistochemically positive for S100, Vimentin (with variable staining), CD56 and in 3 cases also for CD68 and MITF; negative for HMB45, MelanA, AE1/AE3, EMA, and Desmin (Figure 3).

\section{Discussion}

GCT of the breast is a very uncommon condition. Shwannian origin has been found to be the most accepted histogenesis due to immunophenotypical (S100+) and ultrastructural similarities 


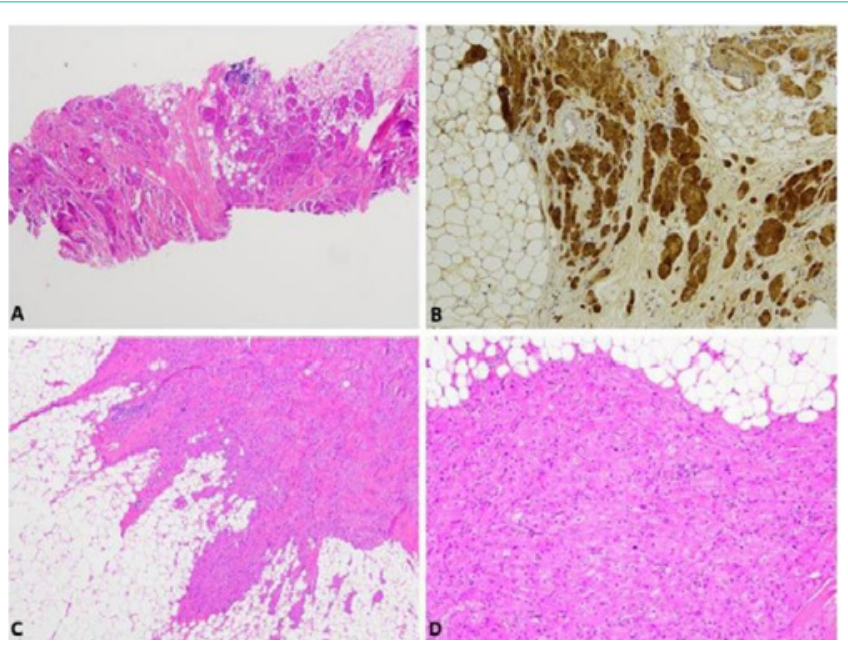

Figure 3: Pathological findings of granular cell tumor. A) Bioptical sample of breast parenchyma occupied by a proliferation of polygonal eosinophilic cells, organized in sheets or ribbons, separated by collagenous bands (H\&E, 4X); B) Neoplastic cells resulted positive for S100 staining (LSAB-HRP, 10X); C) Breast mass with poorly defined margins, composed of sheets and nests of round/polygonal eosinophilic cells (H\&E, 10X); D) Neoplastic cells with a syncitial pattern of growth, dense and vesicular nuclei, with mild to moderate nuclear atypia and abundant eosinophilic cytoplasm with coarse granules (H\&E, 20X).

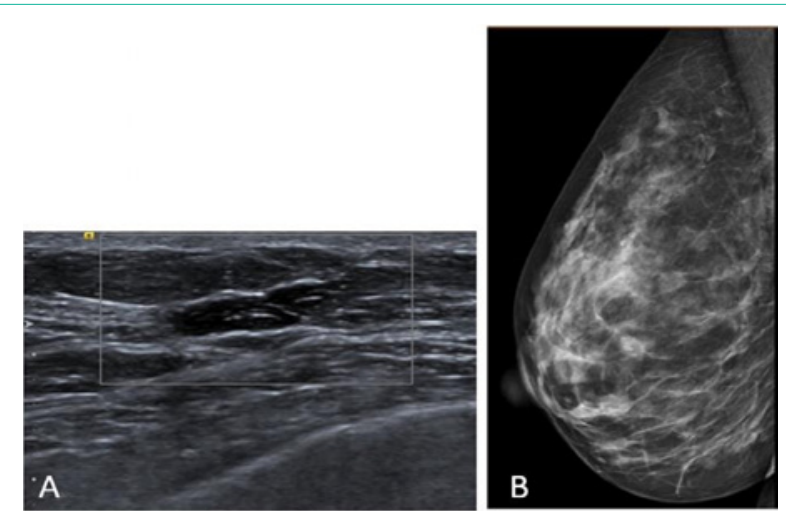

Figure 4: (A) US findings of the lesion. An irregular-shaped mass with slightly uneven internal echo level was observed on US. (B) Mediolateral oblique mammogram shows a subcutaneous opacity with micro calcifications.

exhibited with the Schwann cells.

They arise from the interlobular stroma and the nervous origin is substantiate by the distribution of the cutaneous branches of the supraclavicular nerve, in the upper inner quadrant, where CGTs is most frequently located [6,7]. These tumors are mostly diagnosed in premenopausal women between 30 and 50 years of age, with generally benign biological behaviour and clinical signs similar to breast cancer such as nipple and/or skin retraction.

The radiological presentation on Mammography can also mimic infiltrating breast carcinoma, although they mainly present as a wellcircumscribed mass $[8,11]$. Microcalcifications are not normally a feature of GCTs [12].

On Ultrasound, GCTs may present as solid mass with blurry borders, irregular shape, and attenuated rear echo, or as well- circumscribed solid masses with a more benign appearance [8]. Due to its characteristics similar to classic breast cancer, it requires a careful evaluation and a diagnostic setting aimed at guaranteeing the adequate treatment, in order to avoid unnecessary radical mastectomy and excessive therapies $[13,14]$.

Ultrasound guided percutaneous biopsy of the lesion is the diagnostic procedure of choice. In our series, CGTs of breast usually presented as a painless, firm solid mass, of $1.5 \mathrm{~cm}$ or smaller, with different shapes and features at radiological imaging (Figure 4), and benign histological and cytological aspects.

On MRI this lesion presented low signals in T1-weighted sequences, but are barely in T2-weighted sequences. After contrast, GCT showed both benign and malignant features.

From a pathological point of view GCTs are composed of large cells, characteristically 'clear or eosinophilic' in the granular cytoplasm, due to the accumulation of secretory granules, macroinclusions (pustulo-ovoid bodies of Milian) mitochondria, or lysosomes that are involved in the phagocytosis process of cell membrane in folding [15].

Criteria for atypical and malignant tumors have been described by Fanburg-Smith et al. proposed [16] and include: Increased N/C cytoplasmic ratio, nuclear pleomorphism, tumoral necrosis, spindling of tumor cells, vesicular nuclei with prominent nucleoli, $>2$ mitoses per $10 \mathrm{HPFs}$. A tumor can be considered atypical in presence of 1-2 of the above described features, malignant in presence of $>2$ of these characteristics. Malignant GCTs have a tendency to spread both lymphogenously and hematogenously (lungs).

Other Authors proposed also criteria for granular cell tumor of uncertain malignant potential [17].

In our case series, we have not observed histologically features of malignancy, but we have to be aware that a GCT could be show a malignant clinical behaviour also in presence of a histologically benign diagnosis [18]. The pathological differential diagnosis includes different entities:

- Melanocytic neoplasms, positive for HMB45 and MelanA;

- Leiomuscolar tumors, such as a leiomyosarcoma, being very rare in the breast, positive for desmin and alpha smooth muscle type, negative for S100 and SOX10;

- Alveolar soft part sarcoma, likewise rare in the breast parenchyma, composed of well-defined nests of large polygonal cells with abundant eosinophilic, variably granular cytoplasm, characteristically positive for TFE3.

Finally, in the literature, some cases of infiltrating ductal carcinoma of the breast coexisting with GCT and other lesions that occurs in mastectomy scars have been identified.

It is essential to differentiate between GCT, breast cancer and other pathological entities and although they can have similarities in the clinical diagnosis, the surgical treatment and prognosis are very different [12,19].

Simple local excision with wide margins was the treatment of choice in all cases, also in malignant tumors, where chemotherapy 
and radiotherapy treatments cannot be expected to be effective. Following excision, close clinical surveillance and follow-up with mammography and ultrasonography was the treatment of choice. At the time of writing, no recurrence in all five cases was reported.

\section{Conclusion}

GCT is a rare, usually benign breast disease that can have very similar characteristics to breast cancer both clinically and radiologically. It can be found in different locations of the breast and can have different instrumental characteristics. Histological examination with immunohistochemical analysis is mandatory to make a definitive diagnosis. Treatment of choice consists in wide resection or lumpectomy with margin assessment (no ink on tumor). Sentinel node biopsy is not indicated. No adjuvant therapies are recommended. A long-term follow-up with mammography and ultrasonography is strongly suggested.

\section{Declaration}

Institutional review board statement: The study was conducted according to the guidelines of the Declaration of Helsinki, and approved by the Institutional Review Board and Ethics Committee of Fondazione Policlinico Universitario Agostino Gemelli IRCCS; Rome, Italy.

Informed Consent Statement: Informed consent was obtained from all subjects involved in the study.

\section{References}

1. Ordóñez NG, Mackay B. Granular cell tumor: a review of the pathology and histogenesis. Ultrastruct Pathol. 1999; 23: 207-222.

2. Pieterse AS, Mahar A, Orell S. Granular cell tumour: A pitfall in fna cytology of breast lesions. Pathology. 2004; 36: 58-62.

3. Adeniran A, Al-Ahmadie H, Mahoney MC, et al. Granular cell tumor of the breast: a series of 17 cases and review of the literature. Breast J. 2004; 10 : 528-531.

4. Schrader KA, Nelson TN, De Luca A, Huntsman DG, McGillivray BC. Multiple granular cell tumors are an associated feature of LEOPARD syndrome caused by mutation in PTPN11. Clin Genet. 2009; 75: 185-189.

5. Abrikossoff A. Uber myome ausgehend von der quergesteiften willkurlchen musculator. Virchows Archiv Pathol. 1926; 260: 215-223.
6. Tarallo $\mathrm{M}$, Cigna E, Fino $\mathrm{P}$, et al. Abrikossoff tumor: does it origin in swchann cells? Case report. Ann Ital Chir. 2011; 82: 319-321.

7. Jagannathan DM. Benign granular-cell tumor of the breast: case report and literature review. Radiol Case Rep. 2015; 10: 1116.

8. Gogas J, Markopoulos C, Kouskos E, et al. Granular cell tumor of the breast: A rare lesion resembling breast cancer. Eur J Gynaecol Oncol. 2002; 23: 333-334.

9. Brown AC, Audisio RA, Regitnig P. Granular cell tumour of the breast. Surg Oncol. 2011; 20: 97-105.

10. Miller JA, Karcnik TJ, Karimi S. Granular cell tumor of the breast: Definitive diagnosis by sonographically guided percutaneous biopsy. J Clin Ultrasound. 2000; 28: 89-93.

11. ABREU, Natacha et al. Granular cell tumor of the breast: correlations between imaging and pathology findings. Radiol Bras, São Paulo. 2020; 53: 105-111.

12. Scardina L, Franceschini G, Biondi E, Di Leone A, Sanchez AM, D'Archi S, et al. Myofibroblastoma of the breast: two case reports and literature review. J Surg Case Rep. 2021; 2021: rjab133.

13. Ssi-Yan-Kai G, Barthier S, Trichot C, Prevot S, De Laveaucoupet J. Granular cell breast cancer: a rare misleading lesion. Diagn Interv Imaging. 2015; 96 287-289.

14. Al-Balas M, De Leo A, Serra M, et al. Granular cell tumour of the breast: a rare presentation of a breast mass in an elderly female with a subsequent breast cancer diagnosis. SAGE Open Med Case Rep. 2019; 7: 2050313X19841154.

15. Mittal KR, True LD. Origin of granules in granular cell tumor. Intracellular myelin formation with autodigestion. Arch Pathol Lab Med. 1988; 112: 302303.

16. Fanburg-Smith JC, Meis-Kindblom JM, Fante R, Kindblom LG. Malignant granular cell tumor of soft tissue: diagnostic criteria and clinicopathologic correlation. Am J Surg Pathol. 1998; 22: 779-794.

17. Nasser H, Ahmed Y, Szpunar SM, Kowalski PJ. Malignant granular cell tumor: a look into the diagnostic criteria. Pathol Res Pract. 2011; 207: 164-168.

18. Sonobe H, Iwata J, Furihata M, Moriki T, Ohtsuki Y. Malignant granular cell tumor: report of a case and review of the literature. Pathol Res Pract. 1998; 194: 507-513; discussion 515-516.

19. Scardina L, Franceschini G, Di Leone A, D'Archi S, Santoro A, Mulè A, et al. Low-grade myofibroblastic sarcoma of the breast. Breast J. 2020; 26: 20772078. 